\title{
Research and Application of Simulation-Based Decision-Support
}

\author{
Chun $\mathrm{Yu}^{1, \text { a }}$, Fang Yuan ${ }^{1, \mathrm{~b}}$, Qian Zhang ${ }^{1, \mathrm{c}}$ \\ ${ }^{1}$ Computer and Information Management Center, Tsinghua University, Beijing 100084, China \\ ayuchun@cic.tsinghua.edu.cn, byf@cic.tsinghua.edu.cn, ${ }^{\mathrm{c}} \mathrm{zhq} @ \mathrm{cic} . t$ singhua.edu.cn
}

Keywords: Decision-Support; Decision Support System; DSS Architecture; Simulation; Simulation Model

\begin{abstract}
To find a way to support decision making on basis of simulation, this paper researches the technology of simulation and decision support, and proposes a decision-support method based on simulation, illustrates the actual administrative demand of university, establishes a simulation model, builds the architecture of simulation-based decision-support system, discusses the key technology in the implementation. This module, by summarizing the results of testing and forming the advice of supporting, can well achieve the combination of simulation and decision-support, and thus getting a better result.
\end{abstract}

\section{Preface}

With the deepening of educational informationization, it is more and more difficult for traditional MIS to adapt to the changing internal and external environment, to support new demand on administrative decision making. Decision support system emerges to provide technical support on decision making. Simulation can use models to reproduce the essence process in real environment. DSS based on simulation provides decision maker information to support decision-making by simulating a scheme and analyzing the variable data which is carried from simulation. So, the decision maker can achieve qualitative and quantitative information to support decision-making from the integrated decision support system. Furthermore, decision maker can modify the designed scheme conveniently by the interactive of virtual reality simulation environment after getting the scheme's decision-making information, and can continue simulating and analyzing the new scheme till to get a better scheme.

\section{Research of Simulation-Based Decision-Support}

Simulation. Simulation is a method to establish a mathematical model of system, convert it to the simulation model suitable for computer programming, and carry out simulation experiment on model designed.

Simulation is not a single technology, but a method of solving problems. All models and technologies can be used when modeling a substantive matter and artificial experiments are carried out to find the essence of substantive matter to be solved. Evaluation of various alternatives, through simulation, confirmed the effective solution to the substantive matter. Simulation is a particularly effective way when the system researched is costly, or the experiment is dangerous, or it takes a very long time to learn the consequences caused by different parameters.

An important advantage of simulation is solving those complex problems scarcely solved by analytic method. Some problems are not only difficult to solve analytically, but also to establish mathematical model. Simulation can be used in dynamic process and it seeks best solution using trial-and-error. Compared with the real test, the cost of simulation is relatively low, and the time to get the results is relatively short.

During system simulation, the common way is to verify the hypothesis on basis of what the model designed, and to change the model to be more consistent with real system if necessary. 


\section{1) Simulation model}

Simulation model, whether physical model or mathematical model, is the simulated object's likeness or its structure unit. But not all objects can build a physical model. Computer simulation establish the object's mathematical model first, and then convert it to simulation model suitable for computer processing.

2) Simulation tools

Simulation tools include simulation hardware and simulation software. Computer is the most common component in simulation hardware. Simulation software involves program, package, language, database and system working for simulation. There are many kinds of simulation software, for example, in engineering field, MSC Software, a simulation software used to evaluate system performance, has applied in Aerospace and other high-tech fields more than 45 years.

3) Simulation experiment

Carrying out Simulation experiment can observe the whole changing process of each variable in system model. To seek for the best architecture and parameters of system, experiments are carried out many times. During the system designing, people usually use computer to carry out mathematical simulation experiment for it is convenient and fair to change and convert. During the component developing, half-real simulation experiment can improve the reliability of simulation experiment, using developed real part replace the same part of computer simulation model. During the system developing, half-real simulation experiments are mostly used, to alter each part's or subsystem's structure and parameters.

Decision support system. A decision support system (DSS) is a computer-based information system that supports business or organizational decision-making activities. DSSs serve the management, operations, and planning levels of an organization and help to make decisions, which may be rapidly changing and not easily specified in advance. A properly designed DSS is an interactive software-based system intended to help decision makers compile useful information from a combination of raw data, documents, and personal knowledge, or business models to identify and solve problems and make decisions.

Dialog-Data-Modeling (DDM), the academics most accepted DSS architecture, was proposed by Sprague and Carlson. It indicates DSS is composed of 3 parts: Database Management System (DBMS), Model-base Management System (MBMS), and Dialog Generation and Management System (DGMS).

1) Database Management System (DBMS)

DBMS is a tool to manage database. The database of DSS contains mass of materials, both internal and external. All these materials must be gathered and extracted to turn into information and data structure conductive to decision making, and thus can be used, analyzed, updated and retrieved by users.

2) Model-base Management System (MBMS)

MBMS analyzed information exists inside or outside the database, to integrate all decision models. For example, mathematical measurement mode can be used to analyze and simulate a complicated case, to provide possible plans, and to help user choose one of them. MBMS include modeling language too, give assistance in customizing and building models.

3) Dialog Generation and Management System (DGMS)

The properties possessed by DSS are produced during interacting between DSS and users. The major function of DGMS is managing user interface and implementing interaction. Bennett proposed that DGMS was composed by user, OLAP hardware and software. At meanwhile, the interaction between DSS and user was composed by 3parts too. The first part is action language, means that user communicates with DSS, such as commands that control keyboard, mouse, and so on. The second part is display or presentation language, through which user can see all kinds of output information, such as screen, printer or voice. The last part is knowledge base, contains knowledge user have to learn to use DSS, such as user manual. 
4) Knowledge-based Management System (KBMS)

Because of a lot of unstructured or semi-structured problems, DSS need special knowledge as well as normal function. So besides DBMS, MBMS and DGMS, modern DSS includes KBMS also. It is a very important part. Silverman proposed KBMS should be equipped for supporting the decision flow that mathematical model cannot assist, for helping user to create, use and manage knowledge base, and for integrating expert knowledge base suitable for solving uncertain problems.

Simulation-Based Decision-Support. Computer decision support system(DSS), a computer system employed to provide sufficient information and communication with user in the whole process of decision-making, including data accessing, information processing, scheme evaluating, can assist the user to make a decision scientifically. In the condition of solving practice problems, users often need to obtain a designed scheme's evaluation and optimization information to support decision-making. DSS based on simulation provides decision maker information to support decision-making by simulating a scheme and analyzing the variable data which is carried from simulation. So, the decision maker can achieve qualitative and quantitative information to support decision-making from the integrated decision support system. Furthermore, decision maker can modify the designed scheme conveniently by the interactive of virtual reality simulation environment after getting the scheme's decision-making information, and can continue simulating and analyzing the new scheme till to get a better scheme. The whole process of simulation-based decision-support is shown as Fig.1.

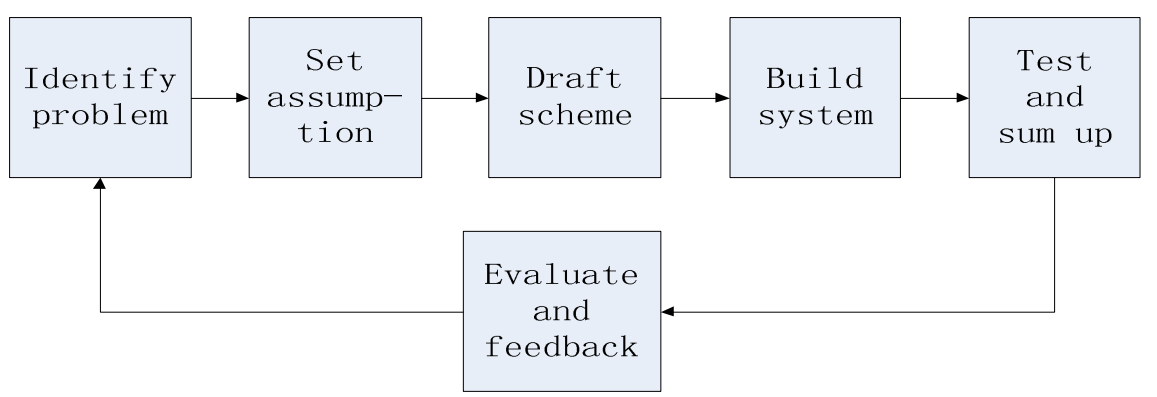

Figure 1. Process of simulation-based decision-support

\section{Application of Simulation-Based Decision-Support of University}

In Tsinghua University, there are 6 classes and 14 sections every day, as shown in Table 1.

Table 1 Class Schedule

\begin{tabular}{|c|c|c|c|}
\hline \multirow{4}{*}{ Morning } & \multirow{3}{*}{$1^{\text {st }}$ class } & $1^{\text {st }}$ section & $8: 00-8: 45$ \\
\cline { 3 - 4 } & \multirow{3}{*}{$2^{\text {nd }}$ class } & $2^{\text {nd }}$ section & $8: 50-9: 35$ \\
\cline { 3 - 4 } Afternoon & $3^{\text {rd }}$ section & $9: 50-10: 35$ \\
\cline { 3 - 4 } & & $4^{\text {th }}$ section & $10: 40-11: 25$ \\
\cline { 3 - 4 } & \multirow{3}{*}{$3^{\text {rd }}$ class } & $5^{\text {th }}$ section & $11: 30-12: 15$ \\
\cline { 3 - 4 } & $4^{\text {th }}$ class & $6^{\text {th }}$ section & $13: 30-14: 15$ \\
\cline { 3 - 4 } & & $7^{\text {th }}$ section & $14: 20-15: 05$ \\
\cline { 3 - 4 } & \multirow{2}{*}{$5^{\text {th }}$ class } & $8^{\text {th }}$ section & $15: 20-16: 05$ \\
\cline { 3 - 4 } Evening & $9^{\text {th }}$ section & $16: 10-16: 55$ \\
\hline \multirow{3}{*}{$6^{\text {th }}$ class } & $11^{\text {th }}$ section & $17: 05-17: 50$ \\
\cline { 3 - 4 } & & $12^{\text {th }}$ section & $17: 55-18: 40$ \\
\cline { 3 - 4 } & & $13^{\text {th }}$ section & $20: 10-20: 55$ \\
\cline { 3 - 4 } & & $14^{\text {th }}$ section & $21: 00-21: 45$ \\
\hline
\end{tabular}


The life and extra-curricular activitiesextra-curricular activities of students and teachers are upset because the 5th class begins at 17:05 and ends at 18:40. So a question comes up: can we shorten the class time under the current teaching arrangement of University? How long the class time will shorten to if we can? Otherwise if we cannot, what are the factors that cause it?

We analyzed and decided to apply simulation-based decision-support to this question, establishing a simulation scheme based upon assumptions and then testing to verify the assumptions.

Simulation scheme. First, we assumed that the teaching arrangement of university works in order when the 11th section was canceled and other sections remained the same. Upon this assumption, we established a simulation scheme then, adopting the concept of time pattern.

Time pattern is an important model in time scheduling. In the university, different courses need different time patterns. Some need teaching whole day or whole week, while some others must teaching 2 days later for students to do homework and digest knowledge they learned last class. Time pattern was designed involving class hours per week, class day of week, class period of day and other items, as shown in Fig. 2.

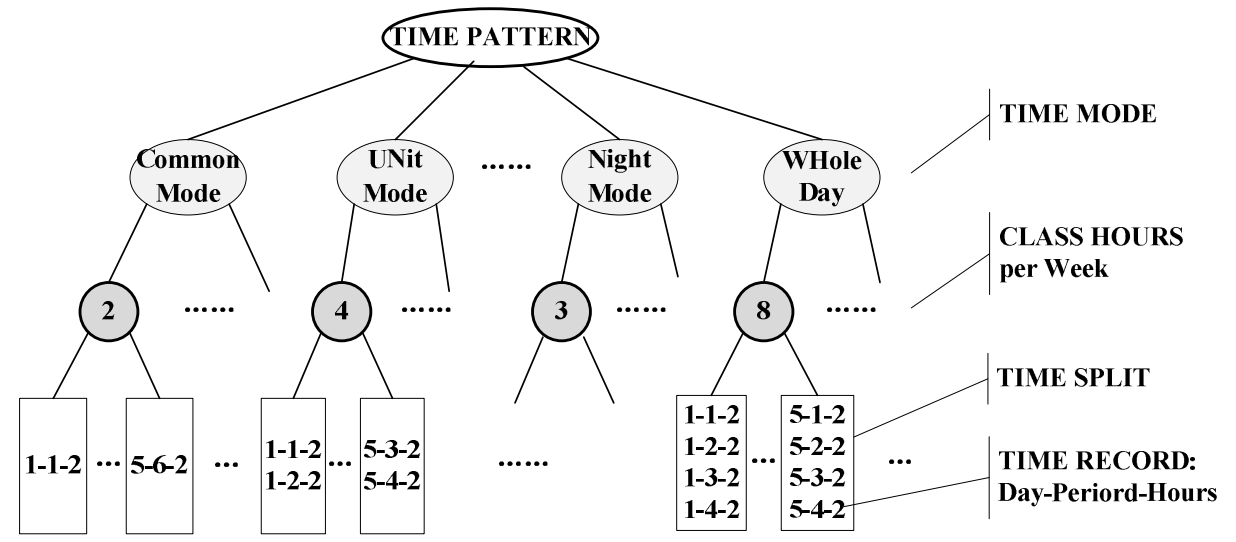

Figure 2. Time Pattern.

We had defined time patterns in advance according to our teaching experience which was used in the course arrangement system. Upon the assumption proposed above, we just reset the time split involving 11th section. The time split before and after resetting are shown in Table 2.

Table 2 Time split reset

\begin{tabular}{|c|c|c|c|c|c|}
\hline \multicolumn{3}{|c|}{ Before resetting } & \multicolumn{3}{|c|}{ After resetting } \\
\hline \multicolumn{3}{|c|}{$7^{\text {th }} \sim 11^{\text {th }}$ sections } & \multicolumn{3}{|c|}{$6^{\text {th }} \sim 10^{\text {th }}$ sections } \\
\hline \multirow{6}{*}{ Afternoon } & \multirow{2}{*}{$3^{\text {rd }}$ class } & $6^{\text {th }}$ section & \multirow{6}{*}{ Afternoon } & \multirow{2}{*}{$3^{\text {rd }}$ class } & $6^{\text {th }}$ section \\
\hline & & $7^{\text {th }}$ section & & & $7^{\text {th }}$ section \\
\hline & \multirow{2}{*}{$4^{\text {th }}$ class } & $8^{\text {th }}$ section & & \multirow{2}{*}{$4^{\text {th }}$ class } & $8^{\text {th }}$ section \\
\hline & & $9^{\text {th }}$ section & & & $9^{\text {th }}$ section \\
\hline & \multirow{2}{*}{$5^{\text {th }}$ class } & $10^{\text {th }}$ section & & \multirow{2}{*}{$5^{\text {th }}$ class } & $10^{\text {th }}$ section \\
\hline & & $11^{\text {th }}$ section & & & $14^{\text {th }}$-section \\
\hline \multicolumn{3}{|c|}{$6^{\text {th }} \sim 13^{\text {th }}$ sections } & \multicolumn{3}{|c|}{$6^{\text {th }} \sim 10^{\text {th }}, 12^{\text {th }} \sim 14^{\text {th }}$ sections } \\
\hline \multirow{6}{*}{ Afternoon } & \multirow{2}{*}{$3^{\text {rd }}$ class } & $6^{\text {th }}$ section & \multirow{6}{*}{ Afternoon } & \multirow{2}{*}{$3^{\text {rd }}$ class } & $6^{\text {th }}$ section \\
\hline & & $7^{\text {th }}$ section & & & $7^{\text {th }}$ section \\
\hline & \multirow{2}{*}{$4^{\text {th }}$ class } & $8^{\text {th }}$ section & & \multirow{2}{*}{$4^{\text {th }}$ class } & $8^{\text {th }}$ section \\
\hline & & $9^{\text {th }}$ section & & & $9^{\text {th }}$ section \\
\hline & \multirow{2}{*}{$5^{\text {th }}$ class } & $10^{\text {th }}$ section & & \multirow{2}{*}{$5^{\text {th }}$ class } & $10^{\text {th }}$ section \\
\hline & & $11^{\text {th }}$ section & & & $14^{\text {th }}$-section \\
\hline \multirow{3}{*}{ Evening } & \multirow{3}{*}{$6^{\text {th }}$ class } & $12^{\text {th }}$ section & \multirow{3}{*}{ Evening } & \multirow{3}{*}{$6^{\text {th }}$ class } & $12^{\text {th }}$ section \\
\hline & & $13^{\text {th }}$ section & & & $13^{\text {th }}$ section \\
\hline & & $14^{\text {th }}$ section & & & $14^{\text {th }}$ section \\
\hline
\end{tabular}


Decision support system. The decision support system architecture in university is shown as Fig. 3 . Material is managed by DBMS, such as information about teachers, students, departments, and courses. Model is managed by MDMS, such as time patterns mentioned above. Knowledge is managed by KBMS, such as constraints and priority-calculating in the course arrangement. All materials, models and knowledge are used in DGMS, which implements the simulation testing function and DSS' interaction with decision maker.

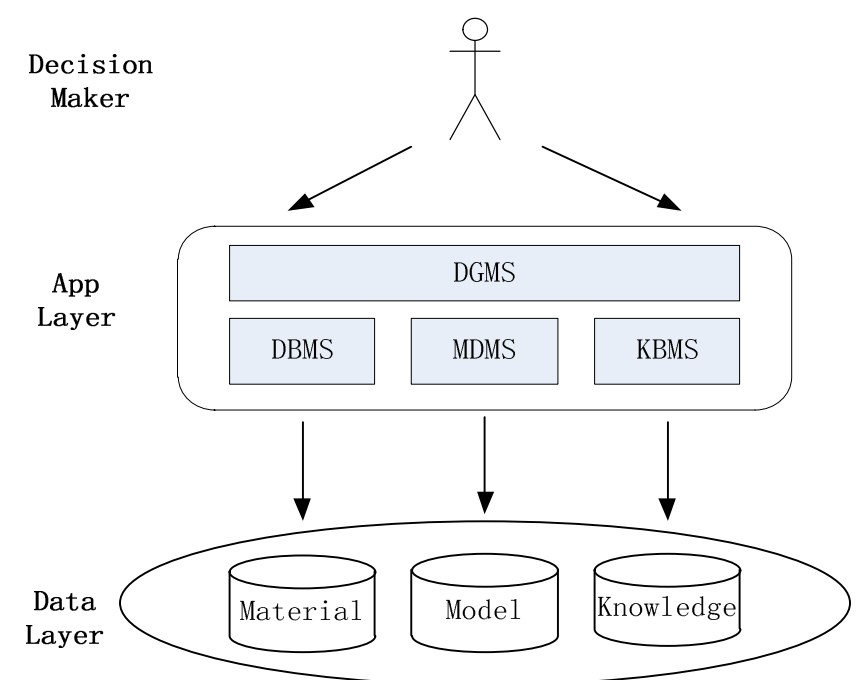

Figure 3. DDS architecture in university

The nature of our problem is to schedule and arranging courses, teachers, students, time and place using simulation scheme. That means the DGMS have to achieve course arrangement functions, solving timetable problem. Timetable problem with large scale and numerous constraints is a NP-hard problem which many researchers in the operational research and computer fields have been dedicated to resolve but have not found a best solution.

The methods to solve timetable problem nowadays are random optimization algorithms, such as simulated annealing algorithm, genetic algorithm, and etc. But these random search algorithms have to check a great many feasible solutions which means huge workload, computational expense, low convergence speed. So we proposed a composite method based on heuristic algorithm, backtracking algorithm and tabu research algorithm.

Heuristic refers to experience-based techniques for problem solving, learning, and discovery. Heuristic methods are used to speed up the process of finding a satisfactory solution, where an exhaustive search is impractical. In more precise terms, heuristics are strategies using readily accessible, though loosely applicable, information to control problem solving in human beings and machines.

Tabu search is a metaheuristic algorithm that can be used for solving combinatorial optimization problems, such as the traveling salesman problem (TSP). Tabu search uses a local or neighborhood search procedure to iteratively move from a solution $\mathrm{x}$ to a solution $\mathrm{x}^{\prime}$ in the neighborhood of $\mathrm{x}$, until some stopping criterion has been satisfied.

Backtracking is an important tool for solving constraint satisfaction problems, for finding all (or some) solutions to some computational problem, that incrementally builds candidates to the solutions, and abandons each partial candidate ("backtracks") as soon as it determines that c cannot possibly be completed to a valid solution.

Backtracking and tabu search are used when a task cannot be arranged successfully. There are two key points in the backtracking and tabu search. First, we do not backtrack to the task former arranged, since it perhaps has nothing to do with the task at the front and maybe we cannot find any available schedule after back and back. Second, we should use limited backtracking to avoid snow slide phenomenon caused by backtracking too deep. 
Simulation testing. Using simulation scheme based upon assumption made above, we rearranged 4 semester courses from 2009 to 2010 academic year, to verify whether the assumption is correct or not. The result is, averagely, $93.5 \%$ courses can be rearranged successfully. The other courses cannot be rearranged are mostly due to 2 factors:

1. Place conflicting. The courses appoint certain classroom when making tasks, and then conflicts during rearrangement. We suggest adjusting these courses' time and place, or increase place resource.

2. Student conflicting. Some students' teaching plan are heavy, and their timetable is full-filled. Thus the course cannot be rearranged if we canceled 11th section. Maybe the teaching plan should be reviewed, and changed if necessary.

\section{Summary}

DSS based on simulation provides decision maker information to support decision-making by simulating a scheme and analyzing the variable data which is carried from simulation. So, the decision maker can achieve qualitative and quantitative information to support decision-making from the integrated decision support system. Furthermore, decision maker can modify the designed scheme conveniently by the interactive of virtual reality simulation environment after getting the scheme's decision-making information, and can continue simulating and analyzing the new scheme till to get a better scheme.

\section{References}

[1] Blischke W R and Murthy D N P, Reliability: Modeling, Prediction, and Optimization, New York, Wiley and Sons, Inc., 2000.

[2] Wang Fei-yue, Computational Experiments for Behavior Analysis and Decision Evaluation of Complex Systems, Journal of System Simulation, 16 (2004) 893-897.

[3] D. C. Montgomery, Design and Analysis of Experiments, 5th Edition. Wiley and Sons Inc., 2000.

[4] Chun Yu, Yanxia Li, Xin Wang, et. al, An Experience-Computer-Based University Course Arrangement System, IEEE PRESS (2011) 388-392.

[5] Wang Fei-yue, On theModeling, Analysis, Control and Management of Complex Systems, Complex Systems and Complexity Science, 3 (2006) 26-34. 